\title{
Using FCM Based Hybrid Computational Approach for Diseases Diagnosis in Traditional Chinese Medicine
}

\author{
T.-C. Chen, P.-S. You, C.-H. Wu, and S.-L. Lin
}

\begin{abstract}
Fuzzy cognitive map (FCM) belongs to one of the soft computing technique for modeling complex systems, which utilize the advantages from the synergistic theories of neural networks and fuzzy logic. The development of FCM highly relies on the human expert experience and knowledge. So, without those from expert(s), the FCM is hard to be constructed successfully. In this study, a self-adaptive FCM without any involvement of experts by using hybrid evolutionary computation approach is proposed. It includes the genetic algorithm (GA) and particle swarm optimization (PSO). The purpose of GA is to decide the significant variables. Based on those variables selected by GA, the most appropriate cognitive map can be constructed by PSO, i.e., the relationship matrix for the set of variables. The purpose of the research is to find the minimum subset of cognitive variables and the corresponding correlation matrix from historical numerical dataset so as to construct the optimal FCM decision model. In this study, the diagnosis of traditional Chinese medicines has been investigated base on twelve-meridian data obtained by meridian energy analysis device. The computational results show that the proposed approach is able to provide higher classification accuracy than those of the approaches in literature or by using commercial software.
\end{abstract}

Index Terms-Fuzzy cognitive map, genetic algorithm, particle swarm optimization, traditional Chinese medicine, twelve-meridian.

\section{INTRODUCTION}

Traditional Chinese medicine (TCM) has been one of the the primary forms of healthcare. Although Western medicine remains the mainstream of the healthcare system, TCM enjoys considerable popularity as a complementary form of healthcare to the Chinese population [1]. In TCM, the meridian energy analysis device (MEAD) is developed by medical experts to objectively measure and acquire the physiological data on the body meridian energy. The integration of the MEAD data with results from Western medical instruments (ex. hematological testing, endoscope, ultrasound, CI scanner and magnetic resonance imaging, etc.) is currently widely employed in TCM clinical practice in order to construct an objectively modern diagnosis.

Manuscript received April 7, 2014; revised May 19, 2014. This work was supported in part by the National Science Council, Taiwan under Grant NSC 101-2221-E-150-058.

T.-C. Chen, C.-H. Wu, and S.-L. Lin are with the Department of Information Management, National Formosa University, Yunlin, 63201, Taiwan (e-mail: tchen@ nfu.edu.tw, melody@ nfu.edu.tw, author@nrim.go.jp)

P.-S. You is now with the Graduate Institute of Marketing and Logistics Management, National Chiayi University, Chiayi, Taiwan (e-mail: psyuu@mail.ncyu.edu.tw).
In real world, the medical diagnosis is highly complicated in nature so that it's not easy to form a comprehensive model taking into account all the significant variables through the conventional statistical methods. Machine learning methods such as neural networks and support vector machines have been displayed to be with more dependable than other conventional approaches. Although the usefulness of using neural networks and support machines has been reported in the literature, the obstacles are in model building and use of model in which the correlation between all the variables are difficulty to be inferred and understood. Recently, cognitive map [2] has been applied in many scientific areas, such as political science [3], organization and strategy planning [4] [5], analysis of business performance indicators [6], software project management [7], supply chain management [8]-[9], medical diagnosis [10]-[12], engineering design [13], etc. When a fuzzy cognitive map (FCM) has been constructed, it becomes a very usefully tool for supporting decision making [14]. For this reason, how to construct an optimal FCM is a significant issue for the research area of decision science. Generally, the manual procedures for developing FCM have occurred, when at least there is one expert who has expertise in the area under study. However, in some cases, the FCM cannot be constructed manually while no expert exists. In the case, the construction of FCM becomes a tough task [15]. Therefore, a systematic way should be developed to overcome the above difficulties. In this study, a hybrid meta-evolutionary approach based on fuzzy cognitive map is proposed to assess abnormal disease data pattern in TCM for constructing the most appropriate fuzzy cognitive map including the predictors, the corresponding correlation matrix (cognitive map) simultaneously so as to building a decision-making model with maximum diagnosis accuracy.

The main purpose of this study is to extract the disease pattern based on the twelve meridians data detected by using the bio-electronic instrument i.e., MEAD. It is hoped that the proposed approach takes account of a refreshing perspective on TCM especially compared to the more common empirical based approach. The proposed approach adopting the GAs and BPSO to assess the various classifications potential is applied for finding the minimum subset of cognitive variables and the corresponding correlation matrix from the historical data so as to construct the optimal FCM decision model. In this approach, each variable can be represented by a gene with a $0 / 1$ value which means the absence or presence of the variable respectively. To each binary chromosome (subset of variables), the BPSO is then acting as a learner to conduct the most appropriate fuzzy cognitive map for a specified category. The proposed approach not only can construct FCM graph topology but also can extract the weight of the edges from historical data simultaneously. 


\section{HYBRID FCM BASED COMPUTATIONAL APPROACH}

Fuzzy cognitive map is one of the soft computing techniques for modeling complex systems, which combines the synergistic theories of neural networks and fuzzy logic. Originally, the development of FCM highly relies on human expert experience and knowledge. So, while the domain knowledge and experience can be not obtained from any of experts, the FCM cannot be constructed successfully either. For this reason, it is to construct a self-adaptive FCM without any involvement of experts by using hybrid evolutionary computation approach. Through the proposed approach the most appropriate FCM decision model can be developed due to the optimal subset of cognitive variables and the corresponding correlation adjacency matrix are obtained simultaneously.

\section{A. Proposed Computational Architecture}

Due to the highly complex computation cost caused by the proposed approach, the grid computing technique has been applied in this study. The computation procedures of the proposed computation approach illustrated in Fig.1 work as follows and the discussion comes in sequence:

\section{Host computer parts:}

Step 1. Randomly generate an initial population of strings which represents the various combination of feature subsets in genetic algorithm.

\section{Client computer parts:}

Step 2. Randomly generate an initial population of particles for the PSO.

Step 3. Activate the FCM inference process and then evaluate each of the particles (solutions) based on the Equation (6) introduced in the following section. The function is to be minimized.

Step 4. Update the values of pi (the best location particle pi has experienced) and pg (the best position of the particle colony) respectively.

Step 5. Modify the velocity of each particle based on the values of pi and pg obtained in step 4 .

Step 6. Modify each particle's position based on its updated velocity so that the position of each particle can be then updated.

Step 7. Check the stopping criterion, if not stop then go to Step 3 Otherwise go to next step.

Step 8. Terminate the solution adjustment process in PSO. The best adjusted solution is then obtained. The best solution of the PSO is then sent to the Host.

\section{Host computer parts:}

Step 9. Check the stopping criterion, if not stop then go to Step 10 Otherwise go to Step 12.

Step 10. Execute the solution improvement by genetic operations including selection, crossover and mutation.

Step 11. When new generation is generated, go to Step 2.

Step 12. End of the process. Input near solutions relation matrix of FCM.

Through the proposed approach, the optimal fuzzy cognitive map (correlation matrix) is obtained to improve the classification accuracy. In our implementation, the subset of decision variables is represented by strings of binary digits of GAs. For each subset of significant features, the corresponding correlation matrix can be constructed by using BPSO. The stopping criterion in the rule mining process is the maximum iterations in this article.

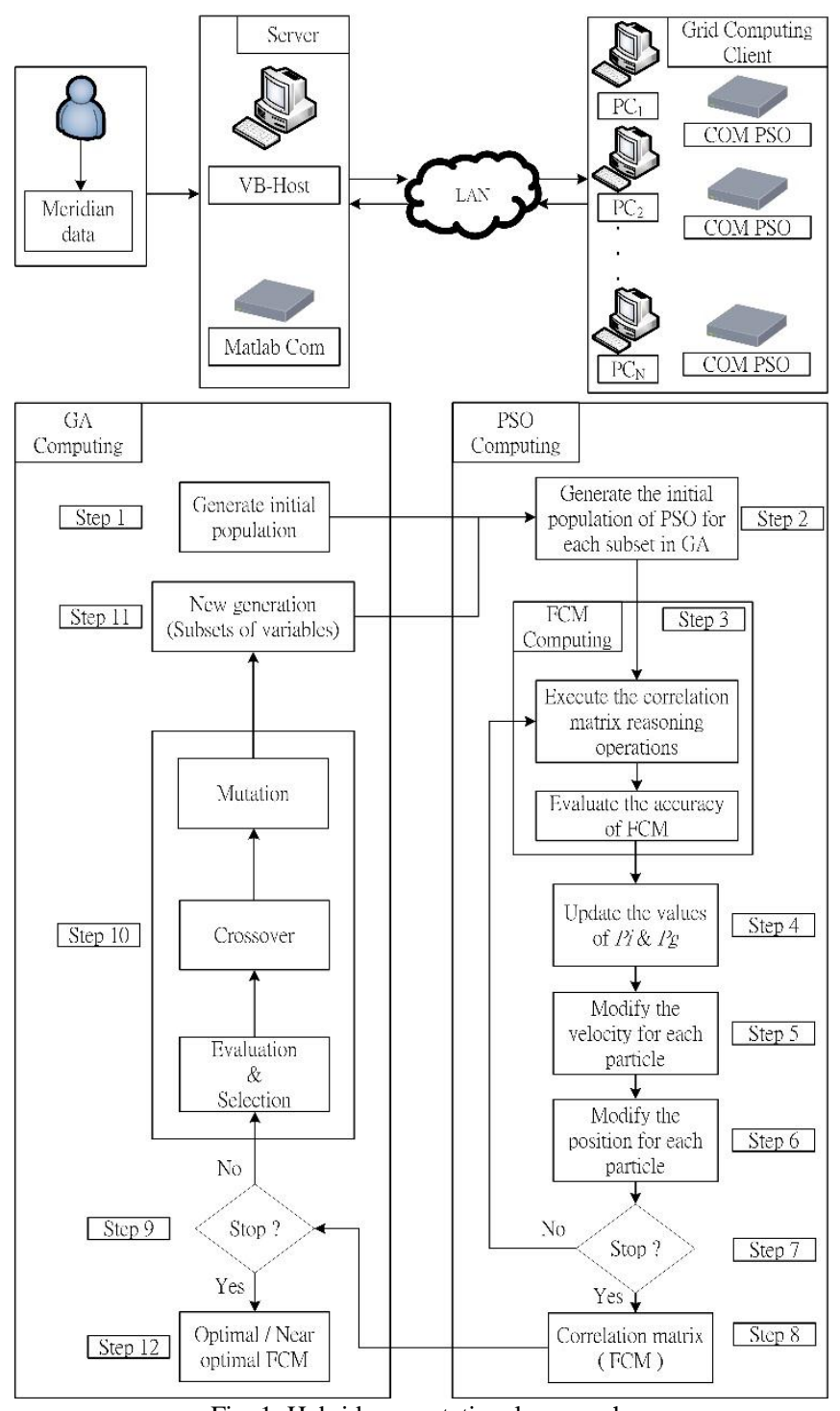

Fig. 1. Hybrid computational approach.

\section{B. Evaluation Function of FCM}

\section{Notation}

$N$ number of variables.

$A_{i} \quad$ the value of concept $i, \forall i=1,2, \ldots, N$.

$t$ computation numbers of FCM, $\forall t=0,1,2, \ldots, K-1$

$E$ relation matrix of FCM, for all $e_{i j}, \forall i=1,2, . ., N ; j=1$, $2, \ldots, N ; i \neq j$.

The computation procedure of FCM can be illustrated by the following equation.

$$
A_{i}^{(k+1)}=f\left(A_{i}^{k}+\sum_{\substack{j \neq i \\ j=1}}^{N} A_{j}^{k} \times e_{j i}\right)
$$

where $A_{i}^{(k+1)}$ is the value of cognitive variable $C_{i}$ obtained by computing it after $k+1$ iterations. $e_{j i}$ is the value of relation between variable $C_{j}$ and variable $C_{i} \cdot f(\cdot)$ is the sigmoid function as follows: 


$$
f(x)=\frac{1}{1+e^{-\lambda x}}
$$

The value of $A_{i}^{(k+1)}$ will become stable (converged) after repeat the computational process continuously. The Eq. (1) \& Eq. (2) can be merged as the following equation.

$$
A_{i}(t+1)=E \times A_{i}(t), \forall t=0, \ldots, K-1
$$

where $A_{i}(t)$ and $A(t+1)$ can be looked as the input and the output of a system model respectively. While $E^{\text {proposed }}$ represents a proposed concept map, we can get the outcome $A_{i}^{\text {estimated }}(t+1)$ after long runs. So, Eq. (3) is interpreted as:

$$
A_{i}^{\text {estimated }}(t+1)=W^{\text {proposed }} \times A_{i}(t)
$$

In Eq. (4), the value of $A_{i}^{\text {estimated }}(t+1)$ is the final results while $E^{\text {proposed }}$ is given. So, when the historical real data $A_{i}^{\text {real }}$ $(t+1)$ is compared with $A_{i}^{\text {estimated }}(t+1)$, the estimation error is then obtained as follows.

$$
\operatorname{Error}_{i}=A_{i}^{\text {estimated }}(t+1)-A_{i}^{\text {real }}(t+1)
$$

Based on Eq. (5), the total errors are then obtained.

$$
\text { Total_Errors }=\sum_{t=0}^{K-1} \sum_{i=1}^{N} A_{i}^{\text {estimated }}(t+1)-A_{i}^{\text {real }}(t+1)
$$

The total errors in Eq. (6) should be minimized.

\section{Mechanism of Solution Representation}

It is noted that the most significant variables must be chosen to form the cognitive map (correlation matrix); otherwise the insignificant variables deteriorate the prediction accuracy and lead to a preposterous outcomes. For finding the optimal set of variables, an appropriate mechanism of variable-set representation in the proposed FCM based approach has to be constructed and designed as a set of binary strings as shown in Fig. 2.

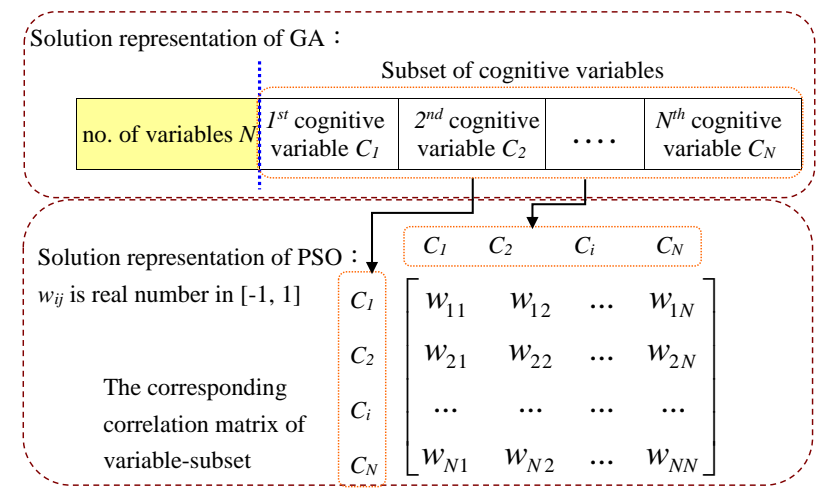

Fig. 2. Solution representation for GAs and BPSO.

After the sets of features being generated by GAs, the BPSO mining process is then launched. For each selected feature in a binary string subset, all the corresponding elements in the correlation matrix (cognitive map) are generated by using BPSO according to the evaluation function in Eq. (6).

\section{Solution Improvement Process}

As shown the mining process in Fig. 1, the genetic operators (crossover operator and mutation operator) are applied to improve the selection of best subset of variables. The crossover operator supplies a thorough search of the sample space to produce better solutions. The mutation operator discharges random perturbations to choose solutions in order to avoid the local optimum. The crossover point is randomly selected and the parent strings are broken into substrings at that point. The offspring are then generated by swapping the substrings of two parents. In the mutation operation, a mutation point is randomly chosen and the binary digit is mutated with a predefined probability. The number of maximum generations is the stop criterion of GA.

\section{EXPERIMENTS AND RESULTS}

To investigate the feasibility and effectiveness of the proposed hybrid evolutionary approach as a response model for the diagnosis in TCM, the meridians of two disease diagnosis data sets are investigated in this study. The proposed hybrid approach is implemented in MATLAB ${ }^{\circledR}$ on the Intel-Core $2 \mathrm{Q} 8300-2.5 \mathrm{GHz}$ PC with setting the parameters for GA and BPSO which has been illustrated in Table I.

TABLE I: THE PARAMETERS SETTING IN THE PROPOSED APPROACH

\begin{tabular}{lll}
\hline \multicolumn{3}{l}{ Parameter Setting } \\
\hline GA & PSO & FCM \\
\hline Population size: 300 & Population size: 300 & Iteration: 20 \\
Max. generations: 300 & Max. generations: 200 & \\
mutation rate: 0.02 & $\mathrm{c} 1: 2$ & \\
crossover rate: 0.95 & $\mathrm{c} 2: 2$ & \\
\hline
\end{tabular}

TABLE II: .NAME OF THE TWELVE MERIDIANS

\begin{tabular}{|l|l|l|}
\hline \multirow{2}{*}{ Twelve Meridians } & \multicolumn{2}{|l|}{ Notations } \\
\cline { 2 - 3 } & $\begin{array}{l}\text { Right } \\
\text { side }\end{array}$ & $\begin{array}{l}\text { Left } \\
\text { side }\end{array}$ \\
\hline 1. Arm Tai Yin Lung Meridian & 1L & $1 \mathrm{R}$ \\
\hline 2. Arm Yang Ming Large Intestine Meridian & 2L & 2R \\
\hline 3. Leg Yang Ming Stomach Meridian & $3 \mathrm{~L}$ & $3 \mathrm{R}$ \\
\hline 4. Leg Tai Yin Spleen Meridian & $4 \mathrm{~L}$ & $4 \mathrm{R}$ \\
\hline 5. Arm Shao Yin Heart Meridian & 5L & 5R \\
\hline 6. Arm Tai Yang Small Intestine Meridian & $6 \mathrm{~L}$ & $6 \mathrm{R}$ \\
\hline 7. Leg Tai Yang Bladder Meridian & 7L & 7R \\
\hline 8. Leg Shao Yin Kidney Meridian & 8L & $8 \mathrm{R}$ \\
\hline 9. Arm Jue Yin Pericardium Meridian & 9L & 9R \\
\hline 10. Arm Shao Yang Triple Burner Meridian & 10L & $10 \mathrm{R}$ \\
\hline 11. Leg Shao Yang Gall Bladder Meridian & 11L & 11R \\
\hline 12. Leg Jue Yin Liver Meridian & 12L & 12R \\
\hline
\end{tabular}

The dataset used in this experiment is provided by the department of Traditional Chinese Medicine in Taichung Hospital, Executive Yuan, Taiwan. This dataset is with 364 patients' records including the measurement of twelve primary meridians. The twelve primary meridians are on the left and right sides of the body. Therefore, the total number of variables is twenty-four and additional one variable, which is 
the response variable representing whether the patient is with disease or not (normal or abnormal). These data were detected by using meridian energy analysis device (MEAD) and summarized in Table II.

For evaluating the performance of the proposed approach, Clementine ${ }^{\circledR}$ from SPSS ${ }^{\circledR}$, a data-mining commercial product, is used to make a comparison with the proposed approach. The C5.0 decision tree algorithm is the core methodology adopted by Clementine ${ }^{\circledR}$ for generating classification rules so that the classification accuracy can be obtained and compared with that of the proposed approach based on the twelve meridians data. It is hoped that the use of the proposed approach is able to provide the scientific analysis for the TCM.

\section{A. Experiment I: Prostate Tumor Dataset}

In the first experiment, the data of prostate tumor used is contained in the twelve meridians dataset mentioned previously. The original dataset contains 212 patient cases with 25 attributes for each case. There are two categories of prostate to be distinguished: normal and abnormal. In the 212 patients' records of the dataset, there are 162 patients $(77 \%)$ with normal condition and the remaining 50 patients $(23 \%)$ with abnormal condition as illustrated in Table III. For evaluating the proposed method, the 10 -fold validation evaluation method is applied for comparing with the results in literatures as shown in Table IV.

TABLE III: PROSTATE DATA

\begin{tabular}{lll}
\hline \hline Category & \# of Cases & Percentage \\
\hline Normal & 162 & $77 \%$ \\
\hline Abnormal & 50 & $23 \%$ \\
\hline Total & 212 & $100 \%$ \\
\hline \hline
\end{tabular}

Based on computational results using the proposed approach in Table IV, it indicates that the proposed method is with highest classification accuracy based on 10-fold validation analysis method. Comparing with other approaches by Chiang [16], support vector machine based software (LibSVM), and the C5.0 decision tree approach in the commercial software SPSS Clementine, the proposed approach is not only with highest accuracy, but also with fewest variables in the subset.

TABLE IV: RESULT COMPARISONS FOR THE PROSTATE DATA

\begin{tabular}{ccc}
\hline \hline Methods & Accuracy $(\%)$ & \# of selected features \\
\hline C5.0 (Clementine, SPSS) & 67.03 & 15 \\
\hline LibSVM & 74.87 & 24 \\
\hline Chiang[16] & 75.89 & 9 \\
\hline Proposed approach & 77.14 & 7 \\
\hline \hline
\end{tabular}

TABLE V: THE CORRELATION MATRIX OF FCM

\begin{tabular}{|c|c|c|c|c|c|c|c|}
\hline & 2R & $8 \mathrm{~L}$ & $7 \mathrm{R}$ & 3L & $4 \mathrm{R}$ & 12L & 9L \\
\hline 2R & 0 & -0.2413 & -0.17423 & -0.23483 & 0.5135 & 0.162225 & 0.112575 \\
\hline 8L & 0.344375 & 0 & 0.6559 & -0.36385 & -0.70275 & -0.95195 & -0.22455 \\
\hline 7R & 0.256425 & 0.882975 & 0 & -0.65938 & -0.2808 & 0.35765 & 0.375325 \\
\hline 3L & 0.048225 & -0.10523 & 0.684 & 0 & -0.10173 & 0.0377 & 0.438 \\
\hline 4R & 0.55305 & 0.170925 & 0.649225 & -0.69743 & 0 & 0.0072 & 0.181725 \\
\hline 12L & 0.10535 & 0.4615 & -0.54648 & 0.145175 & -0.1836 & 0 & 0.22095 \\
\hline 9L & -0.63925 & -0.2102 & 0.6291 & 0.1899 & 0.52665 & -0.63465 & 0 \\
\hline
\end{tabular}

Chinese medicine meridians diagnosis often relies on the subjective tactile experience of the doctors instead of the objective basis. Through the correlation matrix, the significant meridians corresponding to the disease can be investigated more normatively. The best correlation matrix of cognitive map is shown in Table $\mathrm{V}$.

The dimension of this matrix is seven by seven, which indicates the most significant meridians are mined and the corresponding relationships between any pair of the seven meridians can also be observed. Based on the proposed approach, the mined FCM might be the useful auxiliary information to Chinese medicine doctors to have more accurate diagnosis.

\section{B. Experiment II: Cholesterol Dataset}

In the second experiment, there are 360 patients' records. In the dataset, there are 277 patients with normal total cholesterol and the remaining 83 patients with abnormal total cholesterol (see Table VI).

TABLE VI: CHOLESTEROL DATA

\begin{tabular}{lll}
\multicolumn{2}{c}{ TABLE VI: CHOLESTEROL DATA } \\
\hline \hline Category & \# of Cases & Percentage \\
\hline Normal & 277 & $77 \%$ \\
\hline Abnormal & 83 & $23 \%$ \\
\hline Total & 360 & $100 \%$ \\
\hline \hline
\end{tabular}

According to the computational results by applying the proposed approach, which has been summarized in Table VII, it indicates that the proposed method is better than the result obtained by using C5.0 decision tree approach. Again, the classification accuracy is evaluated by using 10 -fold validation analysis method. Comparing with the C5.0 decision tree approach in the commercial software SPSS Clementine, the proposed approach is with higher accuracy and fewer variables in the subset averagely.

\begin{tabular}{ccc}
\multicolumn{3}{c}{ TABLE VII: RESULT COMPARISONS FOR THE CHOLESTEROL DATA } \\
\hline \hline Methods & Accuracy $(\%)$ & \# of selected features \\
\hline C5.0 (Clementine, SPSS) & $67.9 \%$ & 9 \\
\hline Proposed approach & $72.2 \%$ & 7.3 \\
\hline \hline
\end{tabular}

Again, the proposed is with better performance than that of C5.0 decision tree. It indicates the proposed approach is feasible and may provide the valuable diagnosis information to the Chinese Medical Doctor.

\section{CONCLUSIONS}

In this paper, the hybrid meta-evolutionary FCM based mining approach has been proposed to discover the diagnosis cognitive map automatically from the primary meridians data set. By using the proposed approach, the significant predictors with the corresponding matrix of cognitive map are decided by GAs and BPSO to generate the decision model for the classification problems. This may be the first study to use the FCM data mining approach to build a response model by applying primary meridians data in the traditional Chinese Medicine. Based on the extracted correlation matrix of FCM, a response model is then built to discriminate normal data from the abnormal data. The proposed approach not only can construct FCM graph topology but also can extract the weight 
of the edges from historical data in practice. The results of the numerical experiment illustrate that the fuzzy cognitive map obtained by the proposed method have higher classification accuracy than those obtained by using commercial software product or approach in literature. It indicates that the proposed method is a suitable tool for eliciting and representing experts' decision rules and thus it provides effective decision supports for the meridian diagnosis classification problems for Chinese Medical. Further possible improvements may be made by incorporating the grid computing technique and extension to multi-classification mining approach.

\section{REFERENCES}

[1] S. K. Yee, S. S. Chu, Y. M. Xu, and P. L. Choo, "Regulatory control of Chinese proprietary medicines in Singapore," Health policy, vol. 71, no. 2, 133-149, 2005.

[2] R. Axelrod, Structure of decision: the cognitive maps of political elites; New York: Princeton, 1976.

[3] A.K. Tsadiras, I. Kouskouvelis, and K. G. Margaritis, "Using fuzzy cognitive maps as a decision support system for political decisions," Lecture Notes in Computer Science, vol. 2563, pp. 172-182, 2003.

[4] D. Paradice, "SIMON: An object-oriented information system for coordinating strategies and operations," IEEE Transactions on System, Man, and Cybernetics, vol. 22, no. 3, 513-525, 1992.

[5] K.C. Lee and H.S. Kim, "A fuzzy cognitive map-based bidirectional inference mechanism: An application to stock investment analysis," International Journal of Intelligent Systems in Accounting, Finance and Management, vol. 6, no. 1, pp. 41-57, 1997.

[6] D. Kardaras and G. Mentzas, "Using fuzzy cognitive maps to model and analyze business performance assessment," in Advances in Industrial Engineering Applications and Practice II, J. Chen, A. Mital, Eds., pp. 63-68, 1997.

[7] R. Luis, S. Rossitza, and L. S. Jose, "Modeling IT projects success with fuzzy cognitive maps," Expert Systems with Applications, vol. 32, no. 2 , pp. 543-559, 2007.

[8] M. C. Kim, C. O. Kim, S. R. Hong, and I. H. Kwon, "Forward-backward analysis of RFID-enabled supply chain using fuzzy cognitive map and genetic algorithm," Expert Systems with Applications, vol. 35, pp. 1166-1176, 2008.

[9] A. J. C. Trappey, C. V. Trappey, and C. R. Wu, "Genetic algorithm dynamic performance evaluation for RFID reverse logistic management," Expert Systems with Applications, vol. 37, pp. 7329-7335, 2010.

[10] P. R. Innocent and R. I. John, "Computer aided fuzzy medical diagnosis," Information Science, vol. 162, pp. 81-104, 2004.

[11] E. I. Papageorgiou, P. P. Spyridonos, D. Th. Glotsos, C. D. Stylios, P. Ravazoula, G. N. Nikiforidis, and P. P. Groumpos, "Brain tumor characterization using te soft computing technique of fuzzy cognitive maps," Applied Soft Computing, vol. 8, pp. 820-828, 2008.

[12] E. I. Papageorgiou, "A new methodology for decisions in medical informatics using fuzzy cognitive maps based on fuzzy rule-extraction techniques," Applied Soft Computing, vol. 11, pp. 500-513, 2011.

[13] W. P. Cheah, Y. S. Kim, K. Y. Kim, and H. J. Yang, "Systematic causal knowledge acquisition using FCM constructor for product design decision support," Expert Systems with Applications, vol. 38, pp. 15316-15331, 2011

[14] B. Kosko, "Fuzzy Cognitive Maps," International Journal of Man-Machine Studies, vol. 24, pp. 65-75, 1986.

[15] M. Ghazanfari, S. Alizadeh, M. Fathian, and D. E. Koulouriotis, "Comparing simulated annealing and genetic algorithm," Applied Mathematics and Computation, vol. 192, 56-68, 2007.

[16] T. W. Chiang, "Grid computing based meta-evolutionary mining approach as classification response mode," Master thesis in Department of Information Management, National Formosa University, 2008.

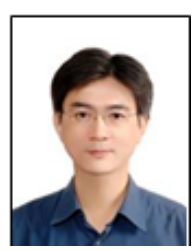

Ta-Cheng Chen received his Ph.D. from the Department of Industrial Engineering, University of Iowa. $\mathrm{He}$ completes his B.Ed. degree in industrial education from National Taiwan Normal University, Taiwan in 1988. Dr. Chen is currently a professor at the Department of Information Management, National Formosa University, Taiwan. His research interest includes data mining, artificial intelligence in optimization, operational research, decision analysis, and clouding computing applications. He published over twenty articles in the field of optimization and data mining by uses of evolutionary computations such as genetic algorithm, artificial immune system, and PSO etc. He also received the excellent teaching award from NFU in 2007 and 2014 respectively.

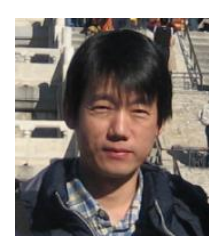

Peng-Sheng You received a Ph.D. degree in management science and engineering from University of Tsukuba, Japan, and his current research interests include decision science, revenue management and supply chain management. He is now a professor at the Department of Marketing \& Logistics/Transportation, National Chiayi University, Taiwan.

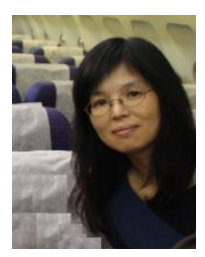

Chun-Hui Wu is an associate professor in the Department of Information Management at National Formosa University, Taiwan. She received her $\mathrm{Ph} . \mathrm{D}$. from the National Yunlin University of Science \& Technology. Her main research lines are data mining, project management and e-learning. Her papers have appeared in academic journals such as Technology Analysis \& Strategic Management, the International Journal of Information Systems and Change Management, and the Journal of International Technology and Information Management.

Shih-Lin Lin received his master degree in management science from the Department of Information Management, National Formosa University, Taiwan in 2012. His research interests include evolutionary computation, and data mining. 